\title{
Modeling injection/extraction in a fracture network with mechanically interacting fractures using an efficient displacement discontinuity method
}

\author{
Alexander Verde and Ahmad Ghassemi* \\ Mewbourne School of Petroleum and Geological Engineering, \\ University of Oklahoma, Norman, OK 73019 \\ (C) 2015. This manuscript version is made available under the Elsevier user license \\ http://www.elsevier.com/open-access/userlicense/1.0/ \\ *Corresponding Author:
}

Tel: +1 4053254347

Email address: ahmad.ghassemi@ou.edu

\begin{abstract}
The displacement discontinuity method (DDM) is frequently used for modeling the behavior of fractures in reservoir modeling. However, the DDM is not computational efficient for large systems of cracks, limiting its application to small-scale situations. Recent fast summation techniques such as the Fast Multipole (FM) Method accelerate the solution of large fracture problems, demanding linear complexity $O(N)$ in memory and execution time with very modest computational resources. In this work we use the FM-DDM method to simulate fracture response while considering fluid flow through the fracture network. This is a novel and efficient approach for solution of large scale coupled fluid flow-geomechanical problem in naturally fractured reservoirs. Several case studies involving fracture networks with several hundred thousands of boundary elements are presented. The results show a good level of accuracy and computational efficiency compared to the conventional DDM. In addition, the approach is shown to be very useful for the design of exploitation strategies in large-scale fracture network situations. The relative positions between injectors and producers and the fracture permeability variation with injection/extraction play an important role on the distribution of stresses in the fracture network, which in-turn, influence the conditions for the fluid-flow such as fluid pressure and fracture permeability.
\end{abstract}

Keywords: Naturally fractured reservoir, displacement discontinuity method (DDM), fast multipole method, fluid flow (FMM), fracture network 


\section{Introduction}

Transient numerical simulations for the coupled fluid flow-geomechanical problem using large number of fracture elements are necessary for realistic representations of unconventional reservoirs for proper design of exploitation strategies. The displacement discontinuity method (DDM) is frequently used for modeling the behavior of natural fractures in linear-elastic rocks [1]. It has been extensively applied in mining and hydraulic fracturing [2-5] due to its semianalytical nature and by reducing the dimensionality of the problem. However, DDM requires computing the influences among all elements so the coefficient matrix of the system of equations is dense and nonsymmetrical. This requirement impacts the computational performance of conventional strategies, either direct or iterative, for the solution of the system of equations when large numbers of elements are involved, making DDM computationally intensive. Direct solvers requires $O\left(N^{2}\right)$ memory and $O\left(N^{3}\right)$ execution time to compute the coefficient matrix and solve a system of equations. On the other hand, iterative methods still need $O\left(N^{2}\right)$ memory but reduce to $O\left(k N^{2}\right)$ the number of operations for convergence in $k$ iterations, assuming $k<<N$ in case of wellconditioned systems. However, large-scale problems with several hundred thousands of unknowns are still beyond the current capability of common personal computers.

Recent fast summation techniques such as the Fast Multipole Method (FMM) can accelerate the solution of large fracture problems, demanding $O(N)$ memory and operations easily accessed from personal computers with modest computational resources [9]. FMM relies on a strategy in which the matrix-vector multiplication is accelerated without forming the coefficient matrix explicitly. This acceleration is carried out by efficiently calculating the interaction between elements using the same DDM discretization but by recursive operations of a quad-tree structure for computation and storage. It permits the solution of larger problems, combining the robustness and accuracy of conventional DDM but with superior performance.

Previous works using DDM and FMM has been limited [6, 7] mainly because of lack of mathematical developments for the fundamental solutions of interest as well as high programming complexity. Peirce and Napier [6] were the first to explore this area introducing a spectral version of the FMM. They proposed a potential representation of the fundamental solutions to approximate the normal and shear displacement discontinuities, applying the method to the granular assemblies problem using up to 1800 boundary elements. On the other hand, Morris and Blair [7] solved the problem of discontinuities within an elastic solid for simulating a 
brittle rock fracture with 22,500 fracture elements, approximating the far-field behavior of the fundamental solutions by known decaying kernels. These studies [6, 7] performed modifications to the DD kernels in order to use the conventional FMM mathematical developments for twodimensional potential problems, impacting accuracy and efficiency. More recently, Verde and Ghassemi [8] developed a Fast Multipole Displacement Discontinuity Methods (FM-DDM) using a kernel-independent version of the FMM [9] which does not requires the implementation of multiple expansions of the underlying kernel to compute geomechanical interactions in naturally fractured reservoirs containing up to 100,000 fractures. In contrast to the cited works which have focused only on evaluating static problems dealing with network response, in this work we consider fluid flow through the fractures, and take into account other relevant geomechanical effects such as non-linear joint deformation. The approach improves upon previous works [3-5] allowing for rapid treatment of large scale coupled fluid flowgeomechanical problem in fractured reservoirs. The solution methodology finds transient changes of fluid pressure and normal and shear displacement discontinuities during injection and production operations accounting for joint deformation. Several case studies involving fracture networks with several hundred thousands of boundary elements are presented to evaluate and compare accuracy and computational efficiency of the approach with the conventional DDM, as well as its potential usefulness for the design of exploitation strategies in large-scale situations.

\section{Mathematical Formulation}

\subsection{Geomechanical model (FM-DDM)}

FM-DDM was developed to compute geomechanical interactions in large-scale fracture network using both the DDM and FFM, respectively, adopting for the latter a kernel-independent version of the classical FMM [9], where analytic multipole and local expansions are not required and using a preconditioned Generalized Minimal Residual Algorithm (GMRES) to solve iteratively the system of equations. In this model for given normal $(n)$ and shear $(s)$ displacement discontinuities (see Fig. 1) in the fractures, defined as the difference in displacement between the two sides of the segment:

$$
D_{i}=u_{i}\left(x_{1}, 0_{-}\right)-u_{i}\left(x_{1}, 0_{+}\right) i=n, s
$$

the normal and shear tractions caused by geomechanical interactions among all fractures can be expressed mathematically as the sum of near and far-field components: 


$$
t_{i}=t_{i}^{\text {near }}+t_{i}^{\text {far }} i=n, s
$$

where the near-field interactions are evaluated as the conventional DDM showing a quadratic, $O\left(N^{2}\right)$, computational complexity, and the far-field influences that involves most of the algebraic products are calculated efficiently using FMM to reduce to $O(N)$ the cost proportional to the number of degrees of freedom $(N)$.

\subsubsection{Computation of the near-field interactions by DDM}

DDM is an indirect BEM for modeling the normal (opening) and shear (ride) displacement discontinuities of fractures embedded in an infinite and elastic medium [1]. The method is based on fundamental or analytical solutions to the problem of a finite segment fracture centered in a Cartesian plane with constant normal and shear discontinuities in displacement. Using DDM, the tractions at a field point $i$ caused by the effect of $m$ fractures at different source locations can be computed as the sum of the contributions of the $m$ fracture segment involved. If accounting for joint deformation [3-5] and shear dilation [3-5] (normal opening caused by shear displacement), the change in time of the traction components to the normal $\left(\Delta t_{n}\right)$ and shear $\left(\Delta t_{s}\right)$ directions can be written as:

$$
\begin{gathered}
\Delta t_{s}^{i}=K_{s}^{i} \Delta D_{s}^{i}+\sum_{j=1}^{m} A_{s s}^{i j} \Delta D_{s}^{j}+\sum_{j=1}^{m} A_{s n}^{i j} \Delta D_{n}^{j}=0 \\
\Delta t_{n}^{i,}=K_{n}^{i}\left(\Delta D_{n}^{i}+\Delta D_{s}^{i} \tan \phi_{d}\right)+\sum_{j=1}^{m} A_{n s}^{i j} \Delta D_{s}^{j}+\sum_{j=1}^{m} A_{n n}^{i j} \Delta D_{n}^{j}=\Delta P_{k}^{i}
\end{gathered}
$$

where $\Delta P_{k}^{i}$ represents the change of the fluid pressure inside a fracture element $i$ in a given time step $k$ and the coefficients $A_{s n}, A_{s s}, A_{n n}$, and $A_{n s}$ represent the global influence containing multiple spatial derivative of the function $f(x, y)$ defined as:

$$
f(x, y)=-\frac{1}{4 \pi(1-v)}\left[\begin{array}{l}
y\left(\operatorname{tg}^{-1} \frac{y}{x-a}-\operatorname{tg}^{-1} \frac{y}{x+a}\right)- \\
(x-a) \ln \left(\sqrt{(x-a)^{2}+y^{2}}\right)+(x+a) \ln \left(\sqrt{(x+a)^{2}+y^{2}}\right)
\end{array}\right]
$$

where $f$ is the relative position function between the element $i$ and $j$ and upon the orientation and length of fracture element $j, G$ is the shear modulus, $v$ represents the Poisson's ratio of the solid medium, and $a$ the fracture half-length. 
On the other hand, joint deformation effects are modeled in Eqs. (3) and (4) using the normal $\left(K_{n}\right)$ and shear $\left(K_{s}\right)$ joint stiffness. Based on the Goodman model [10], $K_{n}$ is estimated via an explicit hyperbolic equation as a function of the initial stiffness $\left(K_{n i}\right)$ and maximum closure $\left(D_{\text {nmax }}\right)$ :

$$
K_{n}=K_{n i}\left(1-\frac{t_{n}^{i,}}{K_{n i} D_{n \max }+t_{n}^{i,}}\right)^{-2}
$$

where $t_{n}^{i}$ is the effective normal traction defined as:

$$
t_{n}^{i}=\sigma_{n}^{i}-P_{k-1}^{i}
$$

with

$$
\sigma_{n}^{i}=\sigma_{x x}^{\infty} \sin \beta_{j}^{2}-2 \sigma_{x y}^{\infty} \sin \beta_{j} \cos \beta_{j}+\sigma_{y y}^{\infty} \cos \beta_{j}^{2}
$$

where $\sigma_{x x}^{\infty}, \sigma_{y y}^{\infty}$, and $\sigma_{x y}^{\infty}$ are the field stress components in $x x, y y$, and $x y$ directions, respectively.

\subsubsection{Computation of the far-field interactions by FMM}

\subsubsection{Brief description of the FMM}

The main idea behind the FMM is to accelerate matrix-vector products $(A x)$ in iterative algorithms without forming the coefficient matrix explicitly, reducing then computation time and saving memory $[11,12]$. Algebraically, the product of the $i$-th row of a $N x N$ matrix $K$ with a column vector $\sigma$ of length $N$ can be expressed just as follow:

$$
f\left\{x_{i}\right\}=\sum_{j=1}^{N} K\left(x_{i}, y_{j}\right) \sigma_{j}
$$

In analogy to Eqs. (3) or (4), Eq. (9) may represent a field value $f$ evaluated at point $x_{i}$ due to the influence of sources (governed by the kernel matrix $K$ ) and located at a set of centers. This computation represents the well-known $\mathrm{N}$-body problem and requires $N \mathrm{x} N$ algebraic products or $O\left(N^{2}\right)$ operations to compute the field values at the set of $N$ evaluation points, [13]. Therefore, the objective of using FMM is to reduce this calculation to ideally $O(N)$ counts by approximating the values of $f$. This approximation is accomplished by classifying the influences into near and far-field interactions, according with the distance among field and source points. The far-field interactions are approximated by concentrating the influence of a cluster of source particles (or fractures in our case) in a single location, assuming that the influences of such 
particles become weaker as the distance between field and source locations increases. This procedure requires to construct a hierarchical tree (or quadtree structure in 2D) to decompose the computational domain and subdivide it at increasing level of refinements, identifiying a near and far sub-domains for each level.

A general algorithm for the far-field calculation in Eq. (9) using the classical version of the FMM involves the following steps: discretization of the boundary, construction of the quadtree structure of the boundary element, upward pass, and downward pass (See Fig. 2). FMM uses the same discretization of the conventional DDM by dividing the boundary of the problem $\Gamma$ into several boundary elements of constant length. Then, a data structure is created by a recursive division of the domain to determine at each level of partition the distribution of boundary elements inside the cells as well as to classify each cell into near and far categories according to the distance to the field points. Following, an upward pass is performed to compute the moments on all cells and at all levels and trace the tree structure upward. Finally, the downward pass translates the influences of the far source points storaged in the cells at different levels to the field points. More details and variations of the FMM have been described previously by other authors $[9,11,14]$.

\subsubsection{Far-field approximation of the traction components using a kernel-independent FMM}

Following Verde and Ghassemi [8], the far-field tractions for elastic rocks are expressed as the sum of several matrix-vector products (denoted as $m v p$ ), following the structure of Eq. (9), for which an FMM approximation is needed for each one. In a compact form, the traction components can be represented mathematically as:

$$
\sigma_{q}^{i}=C \sum_{m=1}^{m v p^{q}}\left(\sum_{j=1}^{N} K_{m}^{i, j} Q_{m}^{j}\right), q=x x, y y, x y ; p=s, n ; i=1,2, \ldots, N
$$

where $C$ is an elastic constant, the subscripts $q$ and $p$ represent the directions of tractions and displacement discontinuities components, and $K_{m}$ and $Q_{m}$ are the kernel matrix and pseudo charge vector associated to the $m$-th matrix-vector product of $m v p_{q, p}$. Initially, the total number of $m v p$ required for $q$ and $p$ directions are twenty-six in total. However, as many $K_{m}$ and $Q_{m}$ are repeated only ten unique FMM approximations with sixteen different pseudo charges are needed. Final expressions of those kernel matrixes and pseudo charges vectors can be found in [8]. Note 
that once estimated the tractions with Eq. (10), a rotation of those components need to the normal and shear directions to be carried out using Eqs. (11) and (12):

$$
\begin{gathered}
\sigma_{s}^{i}=\left(\sigma_{y y}^{i}-\sigma_{x x}^{i}\right) \sin \beta_{j} \cos \beta_{j}+\sigma_{x y}^{i}\left(\cos \beta_{j}^{2}-\sin \beta_{j}^{2}\right) \\
\sigma_{n}^{i}=\sigma_{x x}^{i} \sin \beta_{j}^{2}-2 \sigma_{x y}^{i} \sin \beta_{j} \cos \beta_{j}+\sigma_{y y}^{i} \cos \beta_{j}^{2}
\end{gathered}
$$

where $\beta_{j}$ is the orientation of fracture element $j$ respect the horizontal axis.

\subsection{Fluid-flow model}

The fluid diffusivity equation combines the mass balance equation and Darcy's law and governs the pressure distribution in the fractures [3, 4]:

$$
\frac{k_{f} w_{f}}{\mu} \frac{\partial p}{\partial s}=n w_{f} \Delta L c_{f} \frac{\partial p}{\partial t}-n \Delta L \frac{\partial D_{n}}{\partial t}-q_{s}
$$

where $p, k_{f}$, and $w_{f}$ are the fluid pressure, permeability, and width of the fracture, respectively, $\mu$ is the fluid viscosity, $c_{f}$ is the fluid compressibility (oil, for example), $s$ represents a spatial coordinate along the fracture length, $t$ is the time, $\Delta L$ is the fracture length (or $2 a$ ), $n$ is the ratio of actual fracture void volume $\left(V_{f}\right)$ to the effective fracture void volume for fluid flow $\left(V_{e f}\right)$, and $q_{s}$ is injection or production rate (positive injection) per unit formation thickness.

Eq. (13) represents an additional equation to be solved in conjunction with Eqs. (3) and (4) which account for changes in the fluid density and compressibility, assuming an average aperture of fracture elements, and neglecting the leak-off fluid between the fracture and formation. The fracture permeability $\left(k_{f}\right)$ associated with the normal aperture $\left(w_{f}\right)$ is estimated as:

$$
k_{f}=\frac{w_{f}^{2}}{12}
$$

Using a finite difference scheme in spatial and time, Eq. (13) can be discretized for a given fracture network as follow:

$$
\sum_{j=1}^{m} C^{i j}\left(p^{j}\right)^{k+1}=C^{i i} \frac{\left(p^{i}\right)^{k+1}-\left(p^{i}\right)^{k}}{\Delta t^{k}}-2 a \frac{\left(\Delta D_{n}^{i}\right)^{k+1}}{\Delta t^{k}}-q_{s}^{i}
$$

where $m$ is total fracture elements connected to the $i t h$ fracture, $C^{i j}$ represents the fluid pressure coefficient between elements $i$ and $j$, the subscript $k$ and $k+1$ indicates old and new time levels, and $q_{s}^{i}$ is the injection or production of the ith fracture element. In order to solve Eq. (15), fluid 
pressure and normal displacement discontinuity as well as production or injection rates are set with initial and boundary conditions, respectively. Note that the FMM was not applied for the fluid flow calculation since the effects of fractures which are not directly connected with a particular fracture of reference are excluded. This computation falls into the near-field interactions and requires fracture connectivity information.

\section{Numerical Procedure and Implementation}

The iterative procedure for solving Eqs. (3), (4) and (10) could be summarized as follow. First, the input parameters such as rock properties, initial and boundary conditions, multipole data (number of chebyshev nodes per dimension), and solver's parameters (convergence tolerance, Krylov subspace dimension, and maximum number of restarts) are set. Then, the geometry of the fracture network (i.e., domain dimension, node locations and element connectivity) are assigned. Subsequently, a pre-processing stage is carried out to construct the quad-tree structure of the fracture locations and multipole approximations of selected kernels to efficiently compute via FMM the stresses due to far-field effects of the displacement discontinuities. The next steps involve a computation for each time step to obtain the right-hand side vector (given boundary and initial conditions), preconditioning data (to improve solution convergence) and finally to solve iteratively system of equations by the preconditioned GMRES. For the latter, given a time step at the $k^{\text {th }}$ iteration, the unknown change of the displacement discontinuities $\Delta D_{s}^{k}$ and $\Delta D_{n}^{k}$ and fluid pressure $p^{k}$ in the fractures are assumed. Then, the stress components are computed by summing near and far-field approximations vectors using FMDDM. Lastly, the residual vector is computed after evaluating the fluid flow equation in the fracture network. If the residuals is less than or equal to prescribed tolerance (i.e., $4 \times 10^{-5}$ ), then the stress components, the fracture geometry, and the fracture permeability are updated and the simulation proceeds to the next time step. The accuracy and computational performance are evaluated after the completion of the each time step. The former is done by computing the error between the FMM approach and available analytical or numerical solutions using the Euclidean norm while the latter is evaluated just by recording the required RAM memory and execution time.

Following a previous development (FM-DDM), the couple fluid flow-geomechanical simulator was developed in $\mathrm{C}$ language using NetBeans Platform on Linux, using additionally 
the BLAS/LAPACK libraries in Fortran 77. Again, this new approach uses the GMRES iterative algorithm with a scaling preconditioner to solve the system of linear equations [15]. All cases were run in a Notebook PC with modest computational resources $(2.0 \mathrm{GHz}$ Intel Core 2 Duo T5750 Processor with 4GB of RAM).

\section{Numerical Examples}

This section presents three case studies to evaluate the accuracy of the proposed approach as well as its computational performance under large-scale situations. Table I presents common input properties, FMM parameters and corresponding injection and production data. Note that when more than one injector or producer well is evaluated, the corresponding flow rates are set equal to the total value divided by the number of wells. The iterative GMRES algorithm was set using a tolerance of convergence of $4 \times 10^{-5}$, Krylov subspace dimension of 50 , and 10 as maximum number of restart of the Krylov iteration.

\subsection{Case 1: Model verification}

The aim of this section is to verify the coupled fluid flow-geomechanical model described in Section 2.1. Note that a version of the geomechanical model using FMM has been already verified in a previous work [8] Therefore, a case study involving a linear fracture embedded in an elastic rock and subjected to injection and production wells at both ends is used [16] (See Fig. 3). As transient analytical solutions to predict simultaneous changes of both displacement discontinuities and fluid pressure are not available, inspecting the residual values of the traction and diffusion equations described previously are used. A residual tolerance of 0.001 has been set as a threshold for comparison purposes. Additional qualitatively verifications will be carried out by analyzing some expected patterns of the dynamic pressure and displacements responses to ensure the numerical solution obey the physical processes. Observe that currently the geomechanical model (FM-DDM), were most of the computation are involved, was already verified in a previous work [8] using an analytical solution for constant pressure so most of our attention will be on the pressure computation.

Input parameters are taken from Table I. For this case, the fracture length is divided in 400 boundary elements with $0.072 \mathrm{~m}^{3} / \mathrm{sec}$ as injection and production rates, respectively. Fig. 4 (left) presents the residual mean squared error of the equations for each time step while Fig. 4 
(right) shows the transient response of pressure and displacements at both wells respect to their initial values. Values above (below) zero correspond to the injector (producer) well. Note that the residual errors are significantly smaller than the prescribed tolerance during all the simulation, with a maximum value of $10^{-4}$ for the pressure. On the other hand, it is clear from Fig. 4 (right) that the solution response at both wells shows as expected: i) symmetry pressure pattern respect to the initial value, ii) steady state behavior at late time, iii) null shear displacements, and iv) maximum (minimum) normal displacement discontinuity at the injector (producer), indicating the numerical solution is showing expected behavior.

\subsection{Case 2: Computational performance evaluation}

This case study evaluates memory and operational requirements of the proposed approach under large-scale situations. Figure 5 (left) shows the locations of the injector and producer wells within the fracture network of 66,666 boundary elements (or 200,000 DOFs as every element has associated three unknowns) and Fig. 5 (right) shows the computed fluid pressure distribution for this fracture network. Figures 6 (left) and (right) show the percent of RAM memory and execution time required by the FMM approach and the conventional DDM to perform coupled fluid flow-geomechanical simulations of increasing number of degrees of freedom (DOF), respectively. Table II presents the numerical values of Fig. 6 including the number of iterations for convergence required by the preconditioned version of the program. For additional verification purpose, the relative error of the fluid pressure at the end of the injection between the direct calculation (DDM) and FMM approach is also included. The number of levels $l$ of the quad-tree for the eight (8) cases corresponding to 1000, 2000, 5000, 10,000, 20,000, 50,000, 100,000, and 200,000 DOFs were 2, 2, 3, 3, 3, 4, and 4, 5, respectively.

Figure 6 shows as expected that the memory and execution time employed by the conventional DDM presents a quadratic and cubic behaviour in term of the DOFs. With the available RAM memory (4 GB), DDM needed 21.1\% (0.844 GB) for the 10,000 DOFs case after 28.3 hours of computation, and it is only able to handle a maximum of 20,000 DOFs, estimated by extrapolating the quadratic performance curve.

On the other hand, the proposed simulator showed a linear trend in both memory and

CPU time so larger number of fracture elements can be evaluated. In contrast with the conventional method, the current approach based on the FM-DDM is faster (higher memory- 
efficient) than DDM for problems with more than 1000 (4000) DOFs, needing only $25.7 \%$ of memory and 18.4 hours to solve the largest fracture network problem. Note that in the hypothetical case of doing the same calculation in a personal computer using DDM, such calculation would require $4 \times 100 \mathrm{GBs}$ of RAM and more than 317 years of computation, approximately. Finally, see in Table II that the proposed simulator achieves solution convergence at comparable number of iterations for various problem sizes, showing additionally scalable properties.

\subsection{Case 3: Simulation of a large-scale fluid flow in a deformable naturally fractured reservoir subjected to injection and production operations.}

In this case study, the computational cost, convergence rate, and evolution of flow and geomechanical variables are evaluated. Figure 7 (left) shows the locations of the two injector and four producer wells on the reservoir and Fig. 7 (right) shows the changes of the fluid pressure at the injector and producer wells during the simulation. The naturally fracture network is discretized using a regular mesh of 25,000 boundary elements (75,000 DOFs), exploiting the ability of the approach to solve for larger problems. Formation properties, spatial and time discretization, as well as initial and boundary conditions can be found in Table I. Figure 8 shows the distribution of the fluid pressure in the fracture network at the beginning $(0.047 \mathrm{sec})$ and at the end of the injection and Figs. 9 and 10 show the distributions of the shear displacement discontinuity and fracture width in the network at the corresponding times. Finally, Figs. 11 and 12 show the fracture permeability and the induced stress components $\sigma_{x x}, \sigma_{y y}$, and $\sigma_{x y}$, respectively.

As shown in Fig. 7 (right), the pressure at the injector (producer) wells increase (decrease) with time and reach a steady-state behavior at the end of injection. Therefore, and as expected, higher (lower) values of fluid pressure are found in regions around the injector (producer) wells. Note that at the beginning, these regions are well-separated and their extension limited to fractures around the wells. However, after the fluid injection continues, the connection of these regions is evident forming greater isobaric zones in the fracture network (See Fig. 8). Highest (positive and negative) shear displacement discontinuity (see Fig. 9) are computed in the vicinity of the injector (I1) and producer (P4) wells during the simulation, indicating relevant shear stress concentration at these locations, more likely for being at the corners of the reservoir 
and show the highest pressure changes, with potential to modify the fracture permeability by shear dilation. Note also that when the injection commences, fracture aperture (or negative normal displacement discontinuity) are located mainly near the injector wells, while regions away from this points experiment certain closure in special around the producers (See Fig. 10). At the end the injection, smaller changes in fracture aperture are found not only around the injection points but also in regions between them, clearly connected in a diagonal orientation.

Note that fracture permeability (See Fig. 11) has a pattern similar to the aperture distribution with higher (lower) values around injector (producer) well as expected by Eq. (14). Variations of the spatial gradient apparently depend on several factors such as the relative position between those wells and the induced stresses. The stress distributions are presented in Fig. 12. An analysis of those stress distributions reveals that the gradient of $\sigma_{x x}$ and $\sigma_{y y}$ are orientated in the vertical and horizontal directions, in general showing positive (compression) values in the upper-right zone due to the fluid production (P4) and negative (traction) ones on the lower-left corner as a consequence of the injection (I1). Finally by inspecting the $\sigma_{x y}$ contour plot, it follows the trend of the associated displacement discontinuity (See Fig. 9), presenting higher variations concentrated on the left and lower sides of the fractured reservoir due to the effects of the injection.

\section{Conclusions}

This paper presented a novel approach for an efficient solution of the coupled fluid flowgeomechanical problem in large-scale naturally fractured reservoirs. The DD method is used for modeling the fracture deformation while the finite difference method is used to mode fluid-flow through the fracture. The approach solves for transient changes of fluid pressure and normal and shear displacement discontinuities during injection and production operations in unconventional reservoirs accounting for shear dilation and joint deformation but neglecting fluid leak-off. Several case studies involving fracture networks with several hundred thousands of boundary elements were presented to evaluate and compare the accuracy and computational efficiency of the proposed approach. Results showed higher performance than the conventional DDM, demanding $O(N)$ memory and execution time using modest computational resources and showing its potential usefulness for the design of exploitation strategies in large-scale situations. 


\section{Acknowledgements}

This project was partially supported by the U.S. Department of Energy Office of Energy Efficiency and Renewable Energy under Cooperative Agreement DE-GO18194. This support does not constitute an endorsement by the U.S. DOE of the views expressed in this publication. 


\section{References}

1. Crouch SL, Starfield AM. Boundary element methods in solid mechanics. London: George Allen \& Unwin; 1983.

2. Curran J, Carvalho J. A displacement discontinuity model for fluid-saturated porous media. In: Proceedings 6th International Society for Rock Mechanics Congress, 1987: Montreal.

3. Tao Q, Ehlig-Economides C, Ghassemi A. Investigation of stress-dependent fracture permeability in naturally fractured reservoirs using a fully coupled poroelastic displacement discontinuity model. In: Proceedings SPE Annual Technical Conference and Exhibition, 2009: New Orleans. Paper SPE-124745.

4. Tao Q, Ehlig-Economides CA, Ghassemi A. Modeling variation of stress and permeability In naturally fractured reservoirs using displacement discontinuity method. In: Proceedings 43rd U.S. Rock Mechanics Symposium, 2009, Asheville, North Carolina. Paper ARMA 09-047.

5. Tao Q, Ghassemi A. Simulation of fluid flow In a naturally fractured poro-thermoelastic reservoir. In: Proceedings 44th U.S. Rock Mechanics Symposium, 2010, Salt Lake City. Paper ARMA 10-292.

6. Peirce AP, Napier JAL. A spectral multipole method for efficient solution of large-scale boundary element models in elastostatics. Int. J. Numer. Meth. Eng. 1995; 38(23):4009-4034.

7. Morris JP, Blair SC. An efficient displacement discontinuity method using fast multipole techniques. In: Proceedings 4th North American Rock Mechanics Symposium, 2000, Seattle. Paper ARMA 2000-0959.

8. Verde A, Ghassemi A. Efficient solution of large-scale displacement discontinuity problems using the fast multipole method. In: Proceedings 47th US Rock Mechanics Symposium, 2013: San Francisco. Paper ARMA 2013-717.

9. Fong W, Darve E. The black-box fast multipole method. J. Comp. Phys. 2009; 228(23): 8712-8725. 
10. Goodman RE. Methods of geological engineering in discontinuous rocks. San Francisco: West Pub. Co.; 1976.

11. Greengard L, Rokhlin V. A fast algorithm for particle simulations. J. Comp. Phys. 1987; 73(2): 325-348.

12. Liu Y. Fast Multipole Boundary Element Method. Cambridge: Cambridge University Press; 2009.

13. Buchau A, Rieger W, Rucker WM. Fast field computations with the fast multipole method. Int. J. Comp. Math. Electron. Electric. Eng. 2001; 20(2): 547-561.

14. Ying LX, Biros G, Zorin D. A kernel-independent adaptive fast multipole algorithm in two and three dimensions. J. Comp. Phys. 2004; 196(2): 591-626.

15. Wang H, Yao Z, Wang P. On the preconditioners for fast multipole boundary element methods for 2D multi-domain elastostatics. Eng. Anal. Bound. Elem. 2005; 29(7): 673-688.

16. Lee B. Numerical investigation of fractured reservoir response to injection/extraction using a fully coupled displacement discontinuity method. MS Thesis, 2011, Texas A\&M University.

17. Wang P, Yao Z, Wei Y. FM-BEM evaluation for effective elastic moduli of microcracked solids. Tsinghua Sci Tech 2007; 12(5): 562-566. 
Table I. Common data set used in the numerical examples.

\begin{tabular}{|l|c|}
\hline \multicolumn{1}{|c|}{ Parameter } & Value \\
\hline \hline Domain length, $L,(\mathrm{~m})$ & 400 \\
Shear modulus, $G,(\mathrm{GPa})$ & 15 \\
Poisson's ratio, $v,(-)$ & 0.1 \\
Shear joint stiffness, $K_{s}(\mathrm{GPa} / \mathrm{m})$ & 7.5 \\
Initial normal stiffness, $K_{n i},(\mathrm{GPa} / \mathrm{m})$ & 15.0 \\
Maximum fracture closure, $D_{n \max },(\mathrm{m})$ & $5 \times 10^{-4}$ \\
Far-field stress in $x x$ direction, $\sigma_{x x}^{\infty},(\mathrm{MPa})$ & 15.1 \\
Far-field stress in $y y$ direction, $\sigma_{y y}^{\infty},(\mathrm{MPa})$ & 15.1 \\
Far-field stress in $x y$ direction, $\sigma_{x y}^{\infty},(\mathrm{MPa})$ & 0 \\
Initial fracture aperture, $D_{n}^{0},(\mathrm{~m})$ & $5 \times 10^{-4}$ \\
Initial shear displacement, $D_{s}^{0},(\mathrm{~m})$ & 0 \\
Initial fluid pressure, $p,(\mathrm{MPa})$ & 15 \\
Fluid compressibility, ${ }_{f},(1 / \mathrm{MPa})$ & $5 \times 10^{-5}$ \\
Fluid viscosity, $\mu_{f},(\mathrm{MPa})$ & $5 \times 10^{-9}$ \\
Total injection or production rate, $, q_{s},\left(\mathrm{~m}^{3} / \mathrm{s}\right)$ & $2.16 \times 10^{-4}$ \\
Total simulation time, $t_{s},(\mathrm{~s})$ & $1 \times 10^{6}$ \\
Number of time steps, $n d t,(-)$ & 30 \\
Dilation angle, $\phi_{d},[\mathrm{rad}]$ & 0 \\
Number of Chebyshev nodes per dimension, $n,(-)$ & 5 \\
\hline
\end{tabular}


Table II. Computational performance of the FMM approach and DDM for various problem sizes - Case 2.

\begin{tabular}{|c|c|c|c|c|c|c|}
\hline \multirow[b]{2}{*}{ DOFs } & \multicolumn{2}{|c|}{ DDM } & \multicolumn{3}{|c|}{ FM-DDM } & \multirow{2}{*}{$\begin{array}{l}\text { Error } \\
\times 10^{-12} \\
(\%)\end{array}$} \\
\hline & $\begin{array}{l}\text { Time } \\
(\mathrm{sec})\end{array}$ & $\begin{array}{l}\text { Mem. } \\
(\%)\end{array}$ & $\begin{array}{l}\text { Time } \\
(\mathrm{sec})\end{array}$ & Mem.(\%) & $\begin{array}{l}\text { No. } \\
\text { Iter. }\end{array}$ & \\
\hline 1000 & 51 & 0.2 & 53 & 3.1 & 245 & 13.51 \\
\hline 2000 & 589 & 0.9 & 112 & 3.2 & 286 & 5.23 \\
\hline 5000 & 12,090 & 4.6 & 448 & 3.6 & 326 & 3.12 \\
\hline 10,000 & 102,000 & 21.1 & 1010 & 3.9 & 400 & 0.82 \\
\hline 20,000 & - & - & 3777 & 5.2 & 476 & - \\
\hline 50,000 & - & - & 11,463 & 7.1 & 699 & - \\
\hline 100,000 & - & - & 38,110 & 8.5 & 878 & - \\
\hline 200,000 & - & - & 65,690 & 25.7 & 1043 & \\
\hline
\end{tabular}




\section{List of Figures}

Fig. 1. Fracture segment embedded in a two-dimensional and infinite medium showing constant normal and shear discontinuity displacements [1].

Fig. 2. Principal operations of the FMM [17].

Fig. 3. Linear fracture configuration subjected to fluid injection and production used to verify the FMM approach - Case 1.

Fig. 4. Mean values of the residual equations for each time step (left) and transient response of pressure and displacements at the injector and producer wells (right) - Case 1.

Fig. 5. Locations of the injector and producer wells (left) and fluid pressure distribution (MPa) at the end of injection (right) in the fracture network of 200,000 DOFs used to evaluate the performance of the FMM approach - Case 2.

Fig. 6. Comparison of the memory usage (left) and CPU time (right) between DDM and the proposed FMM approach for increasing numbers of DOFs - Case 2.

Fig. 7. Locations of the injector and producer wells in the fracture network (left) and fluid pressure changes at the wells (right) during the simulation - Case 3 .

Fig. 8. Distribution of the fluid pressure in the fracture network computed at the beginning (left) and at the end (right) of injection - Case 3.

Fig. 9. Distribution of the shear displacement discontinuity in the fracture network computed at the beginning (left) and at the end (right) of injection - Case 3 .

Fig. 10. Distribution of the fracture width in the fracture network computed at the beginning (left) and at the end (right) of injection - Case 3.

Fig. 11. Distribution of the fracture permeability in the fracture network computed at the beginning (left) and at the end (right) of injection - Case 3.

Fig. 12. Distribution of the induced stresses $\sigma_{x x}$ (top), $\sigma_{y y}$ (middle), and $\sigma_{x y}$ (bottom) in the fracture network computed at the beginning (left) and at the end (right) of injection - Case 3 . Units are in $\mathrm{kPa}$. 


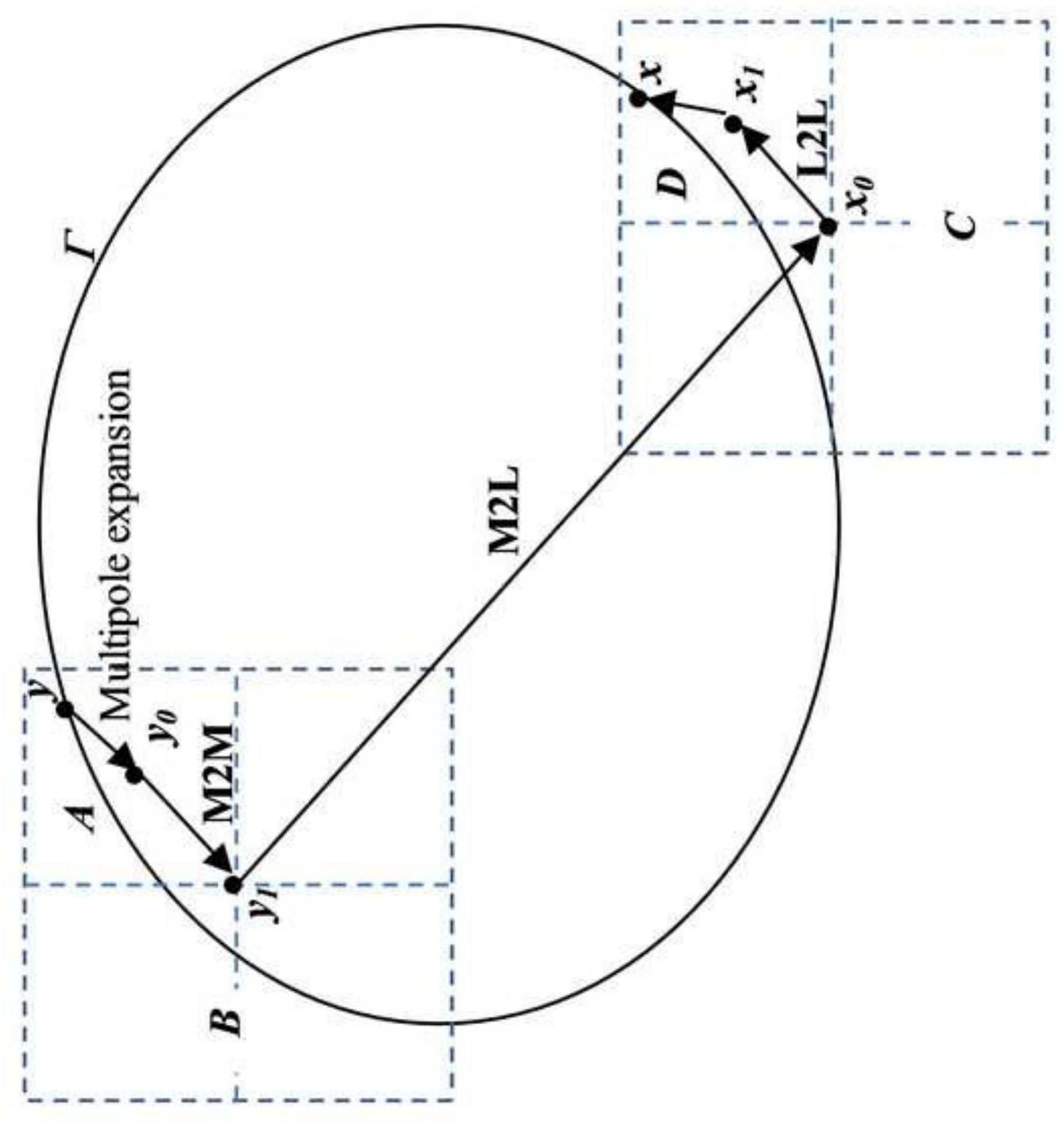

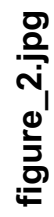




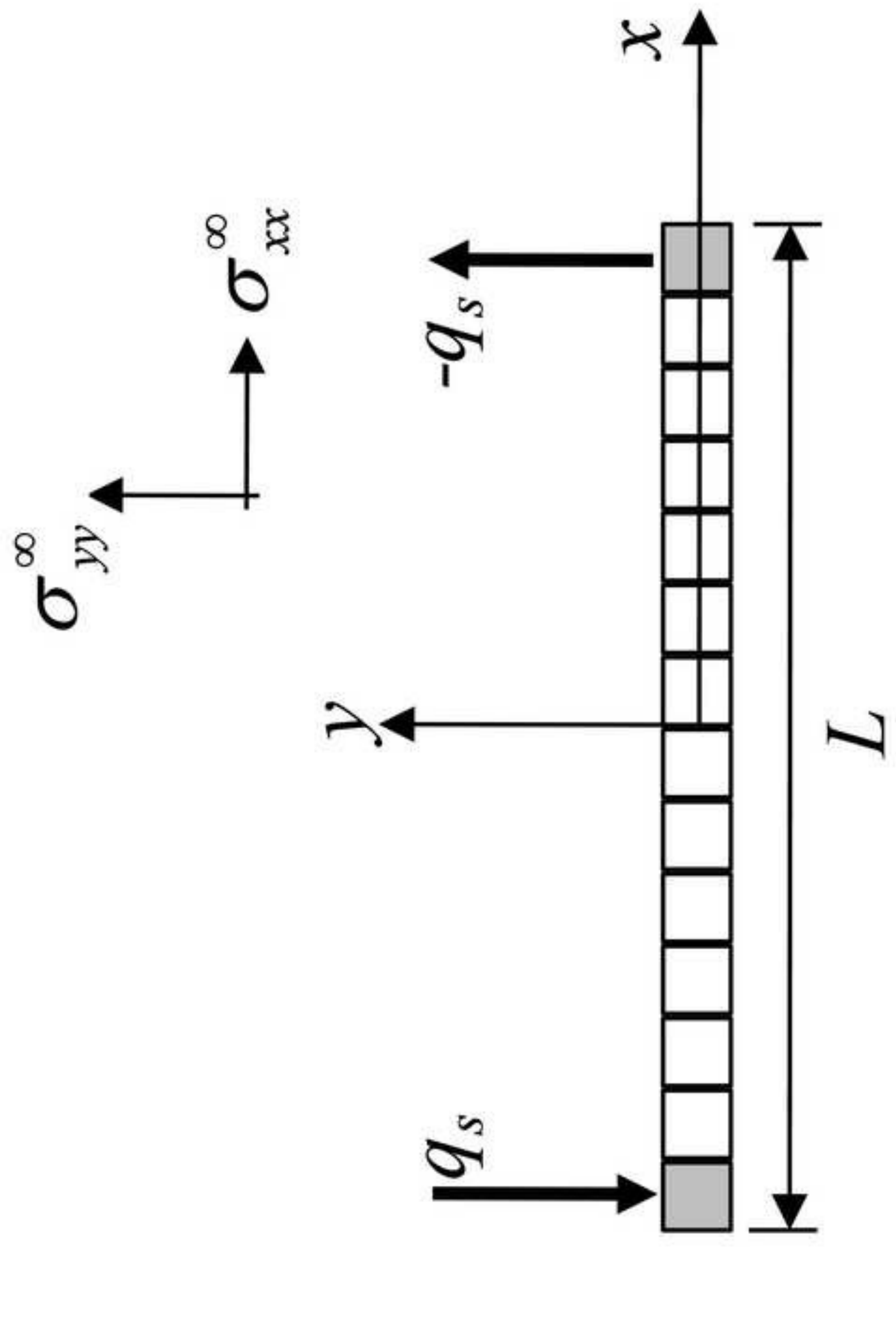




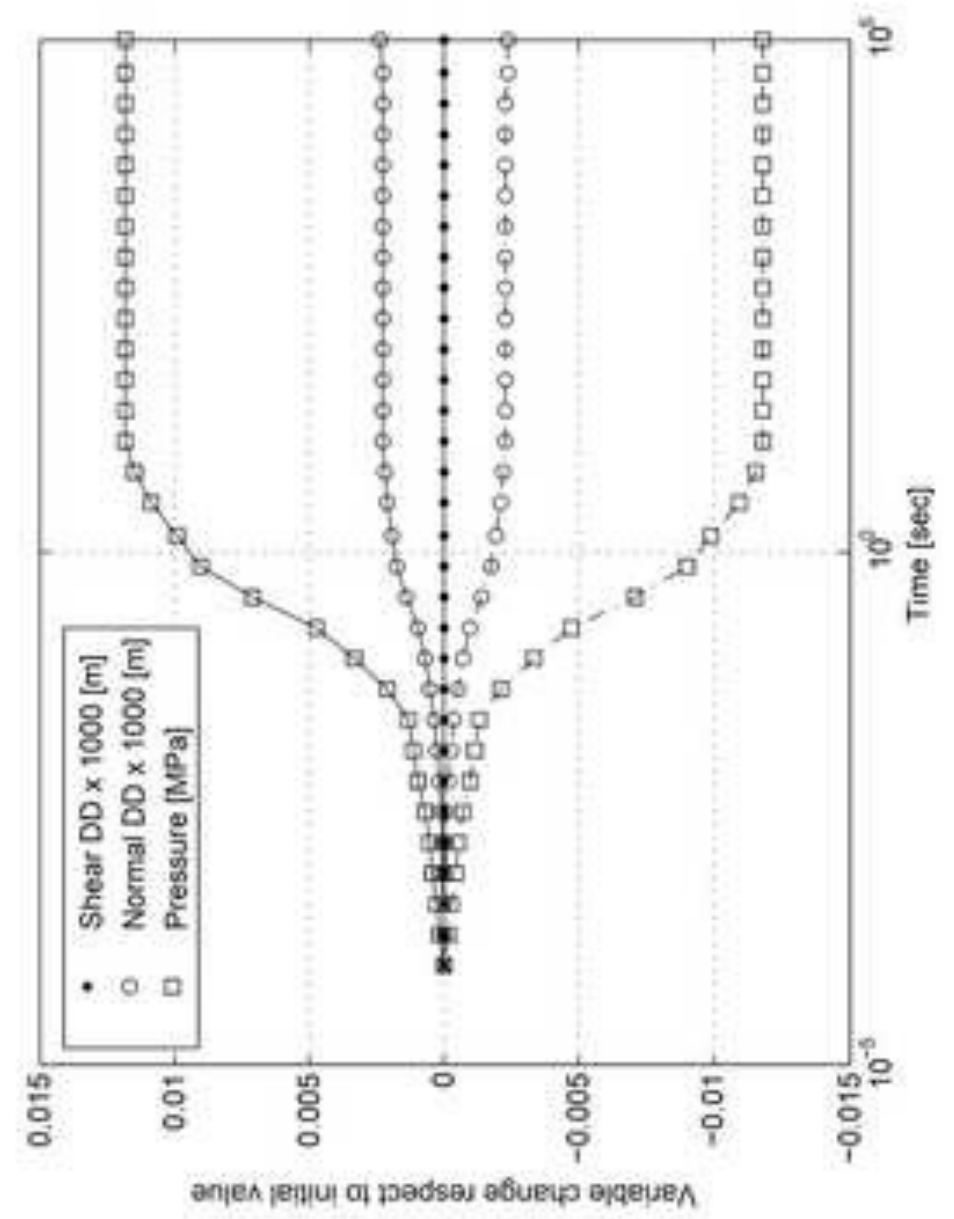

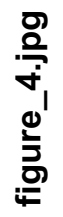

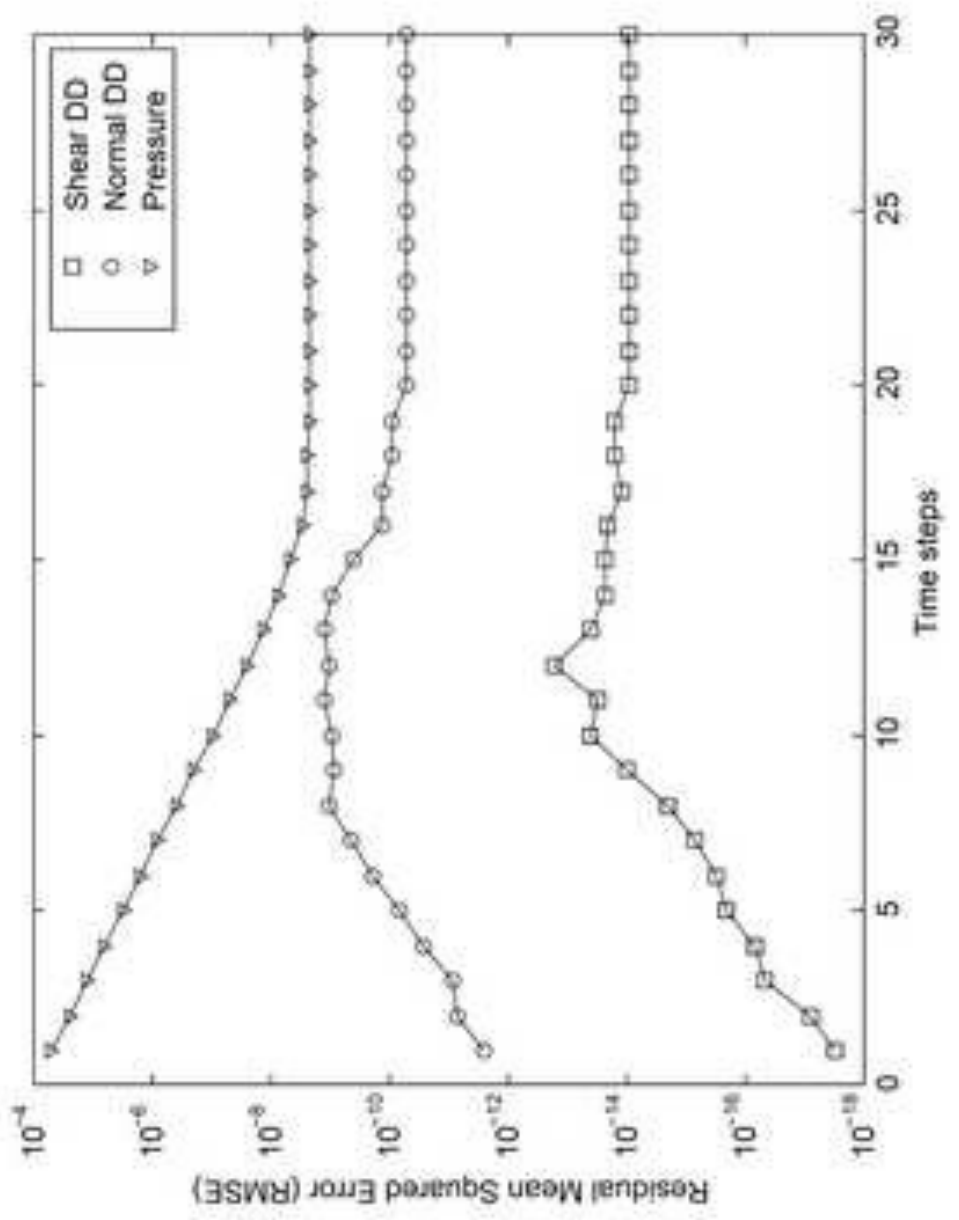




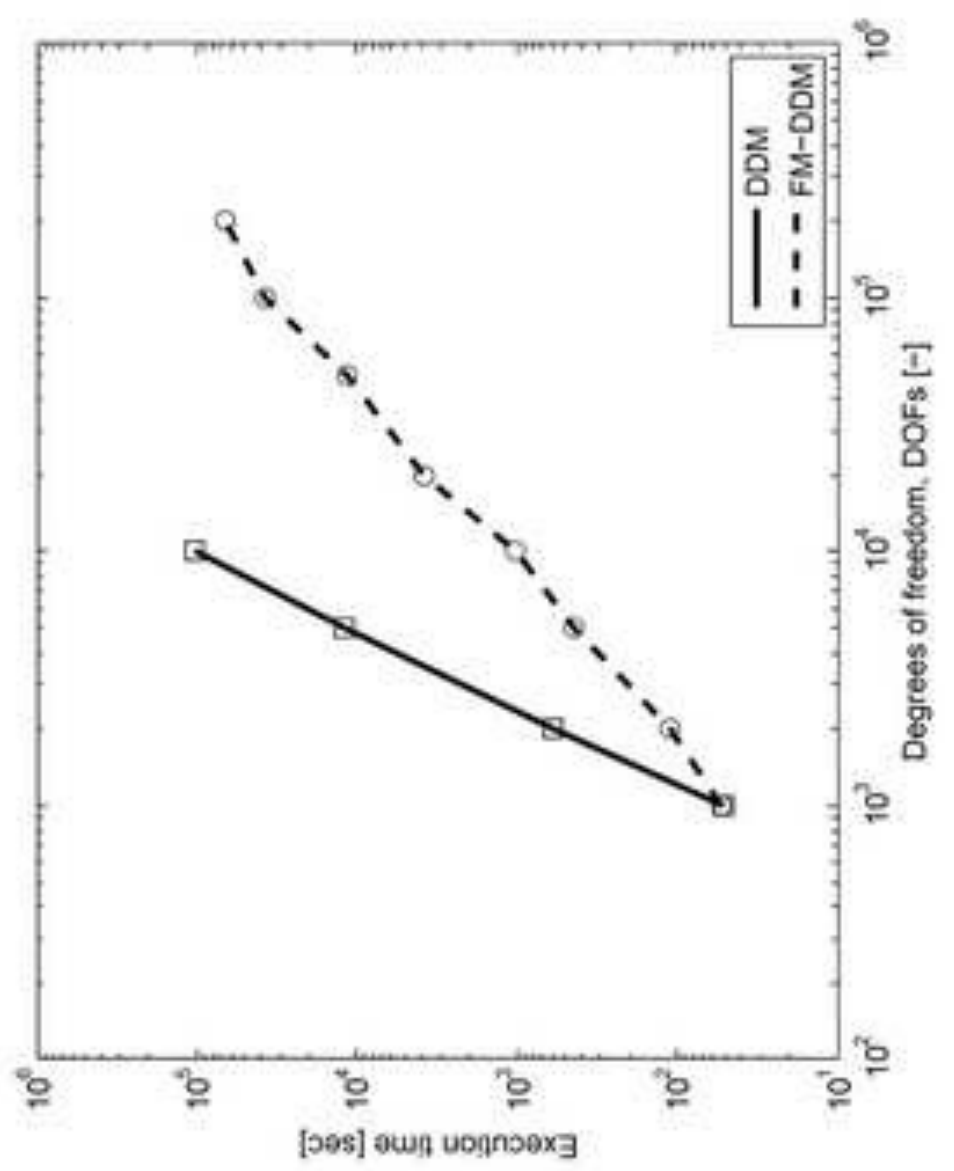

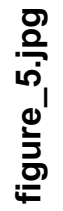

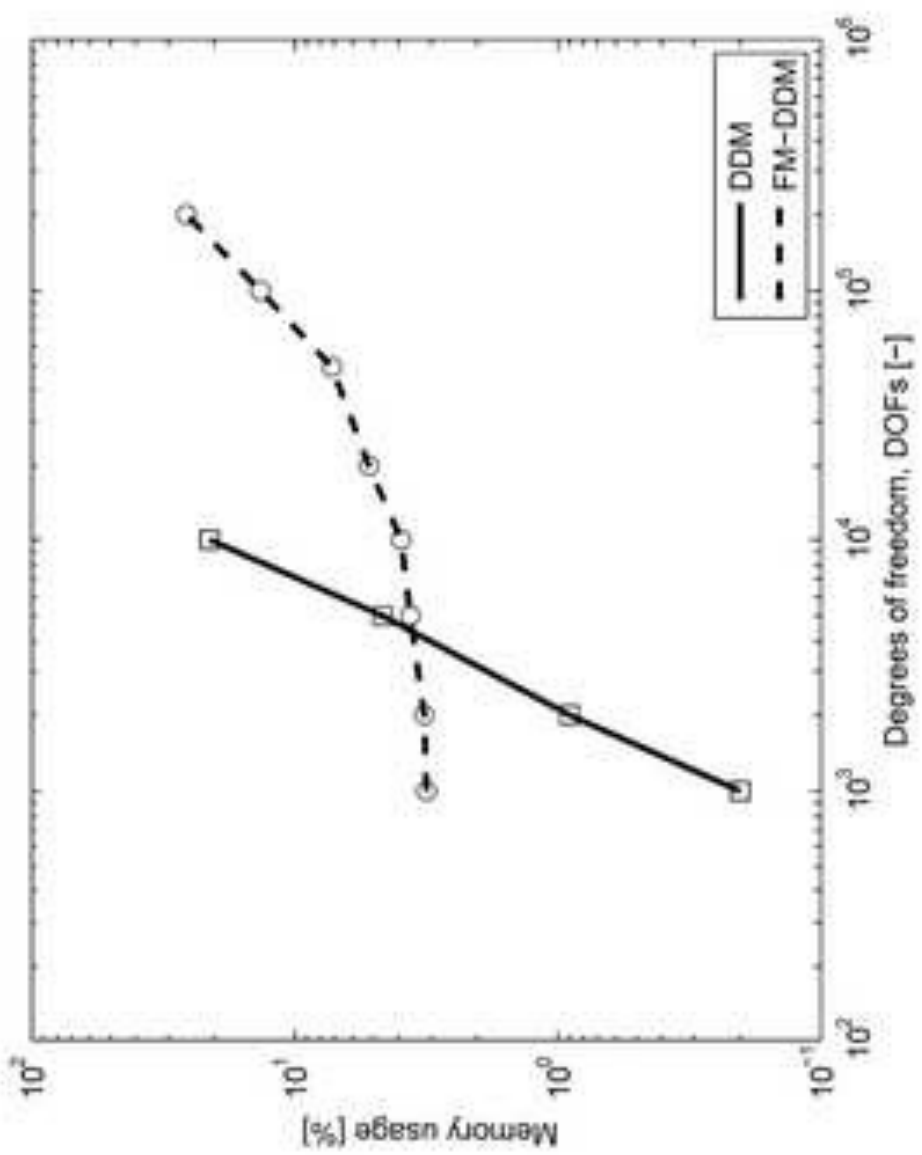



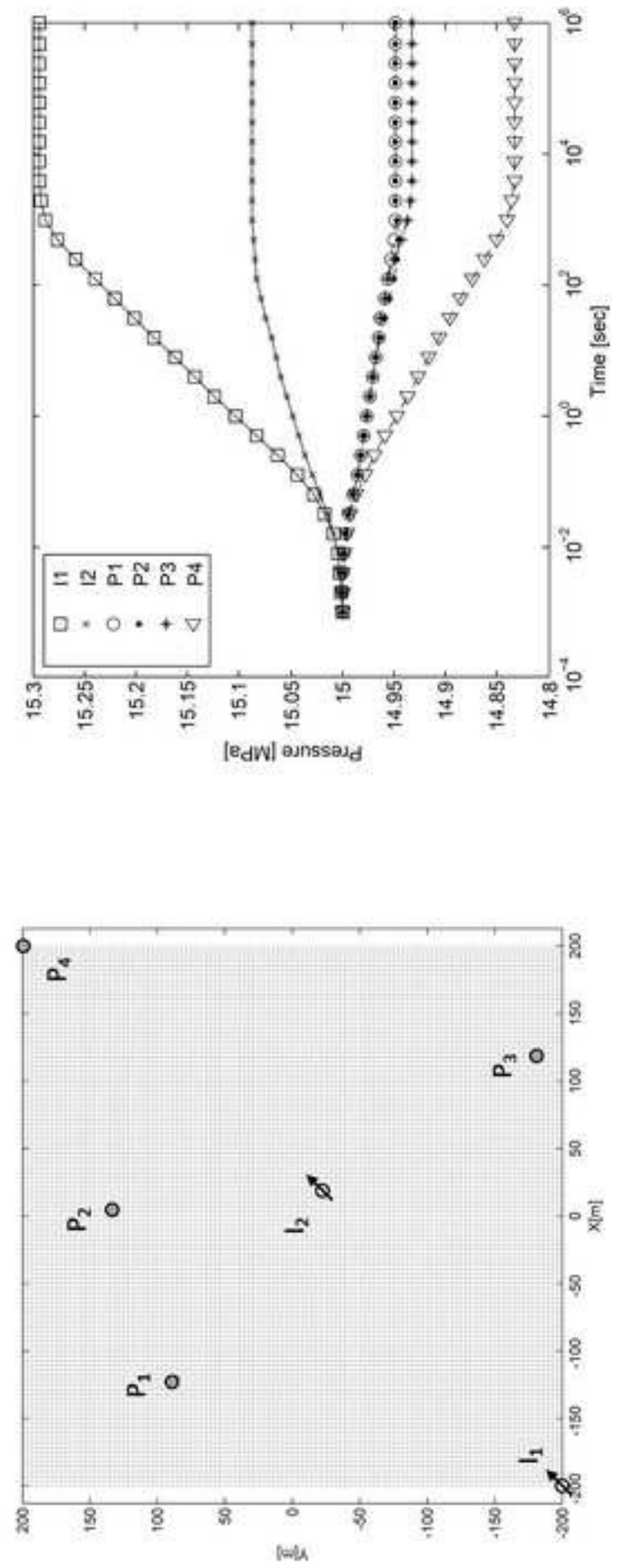

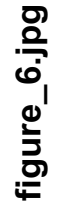



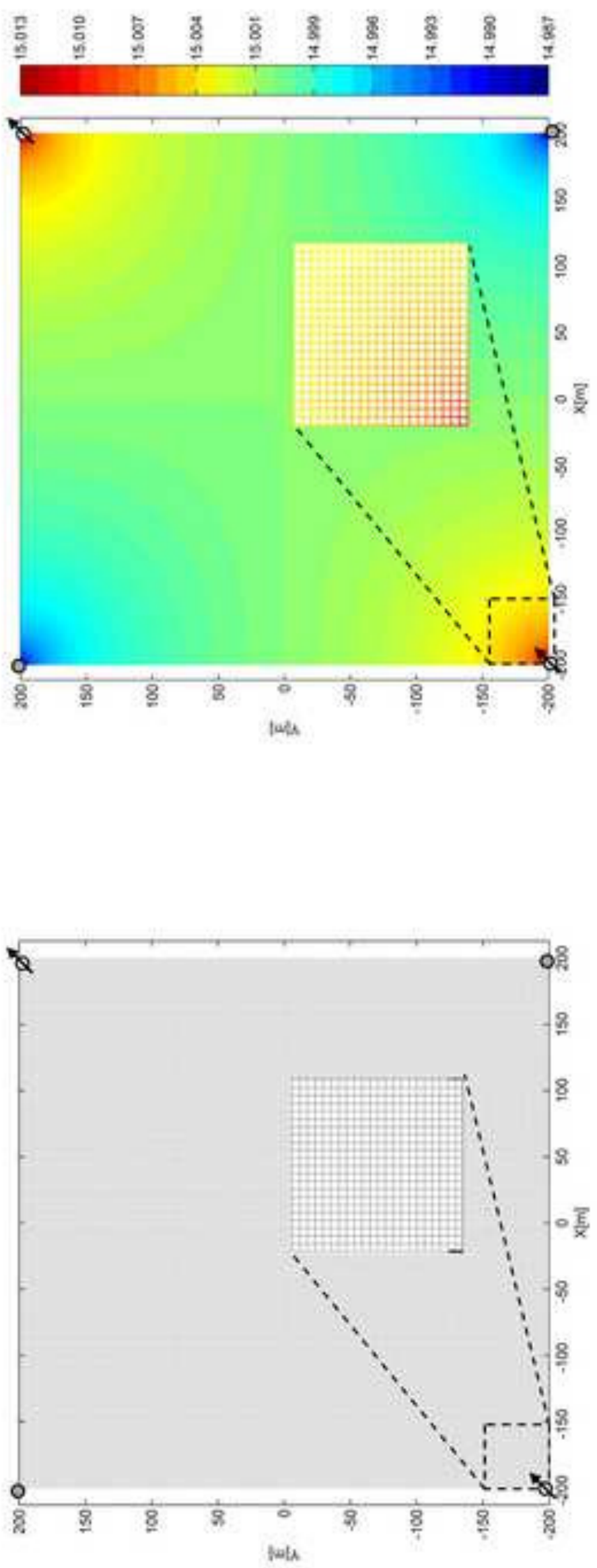

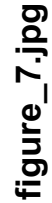



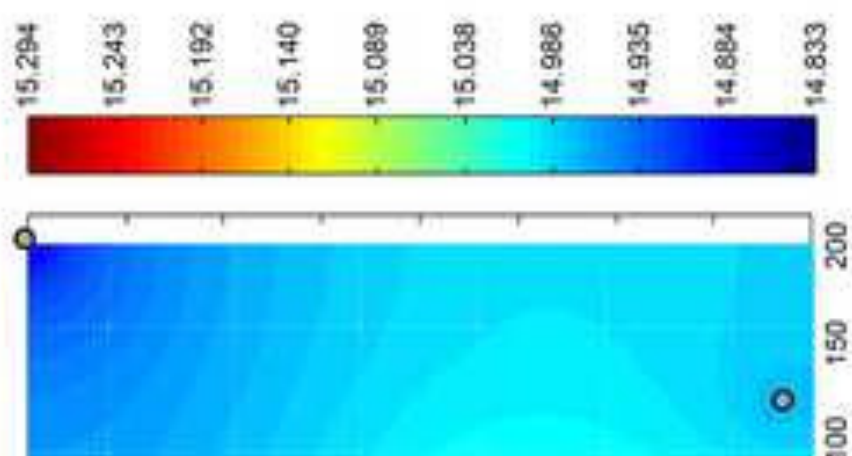

$\frac{\circ}{\circ}$

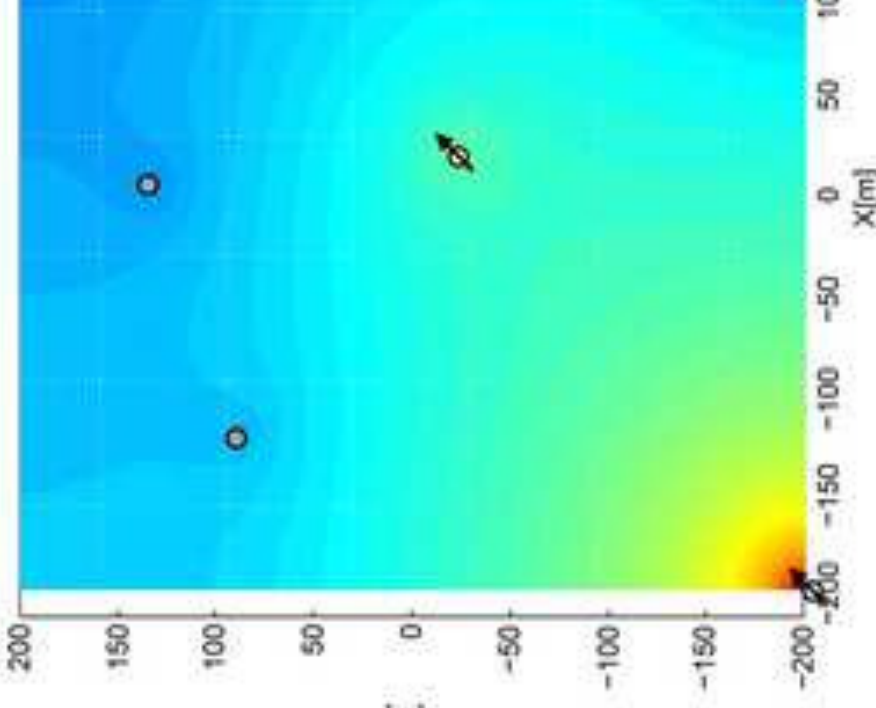

[w]A
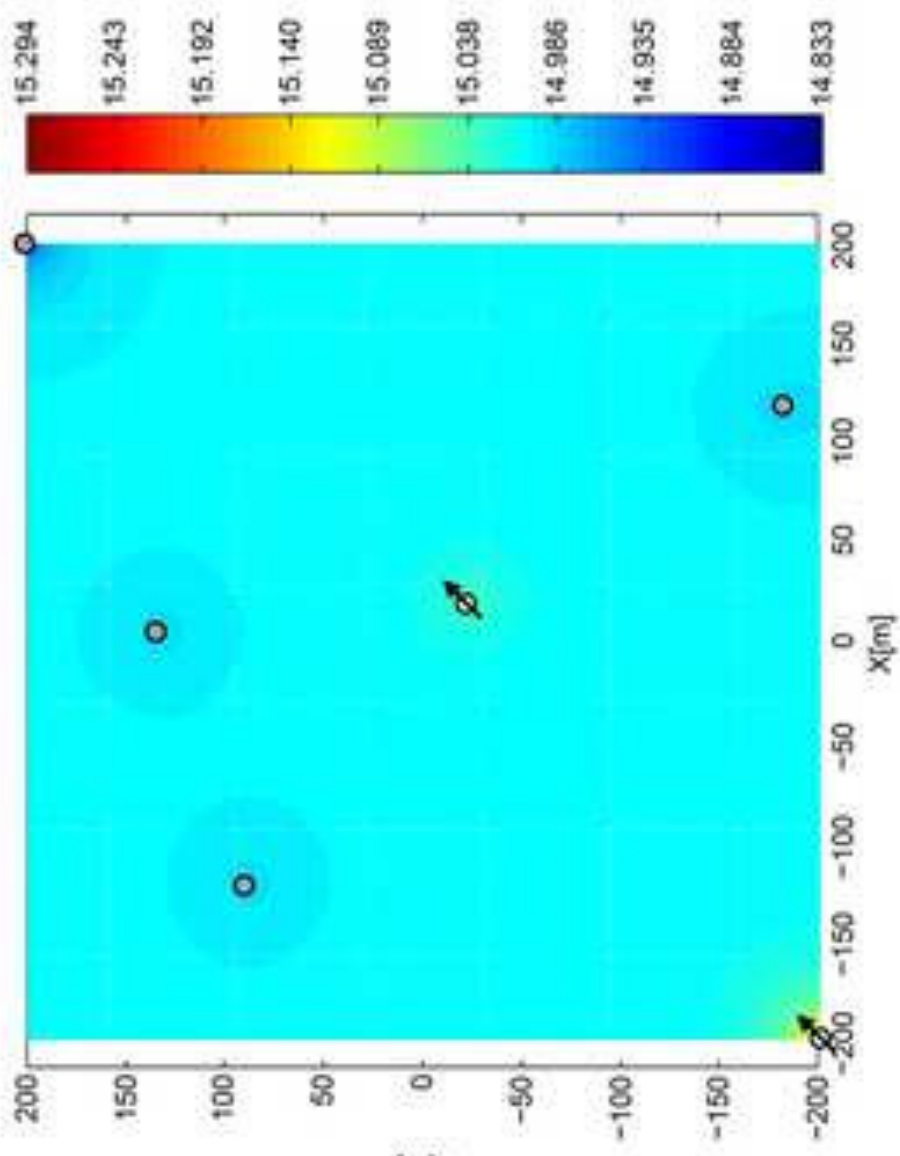

[w] 

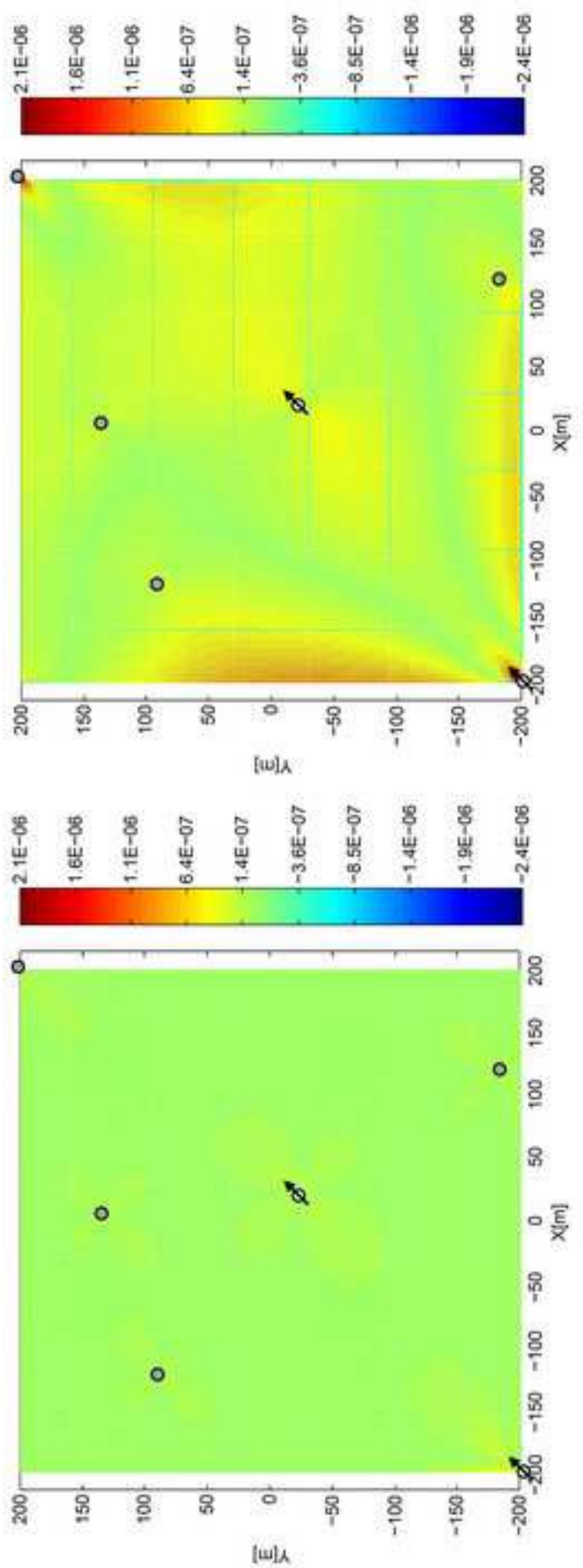

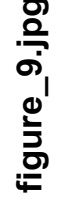




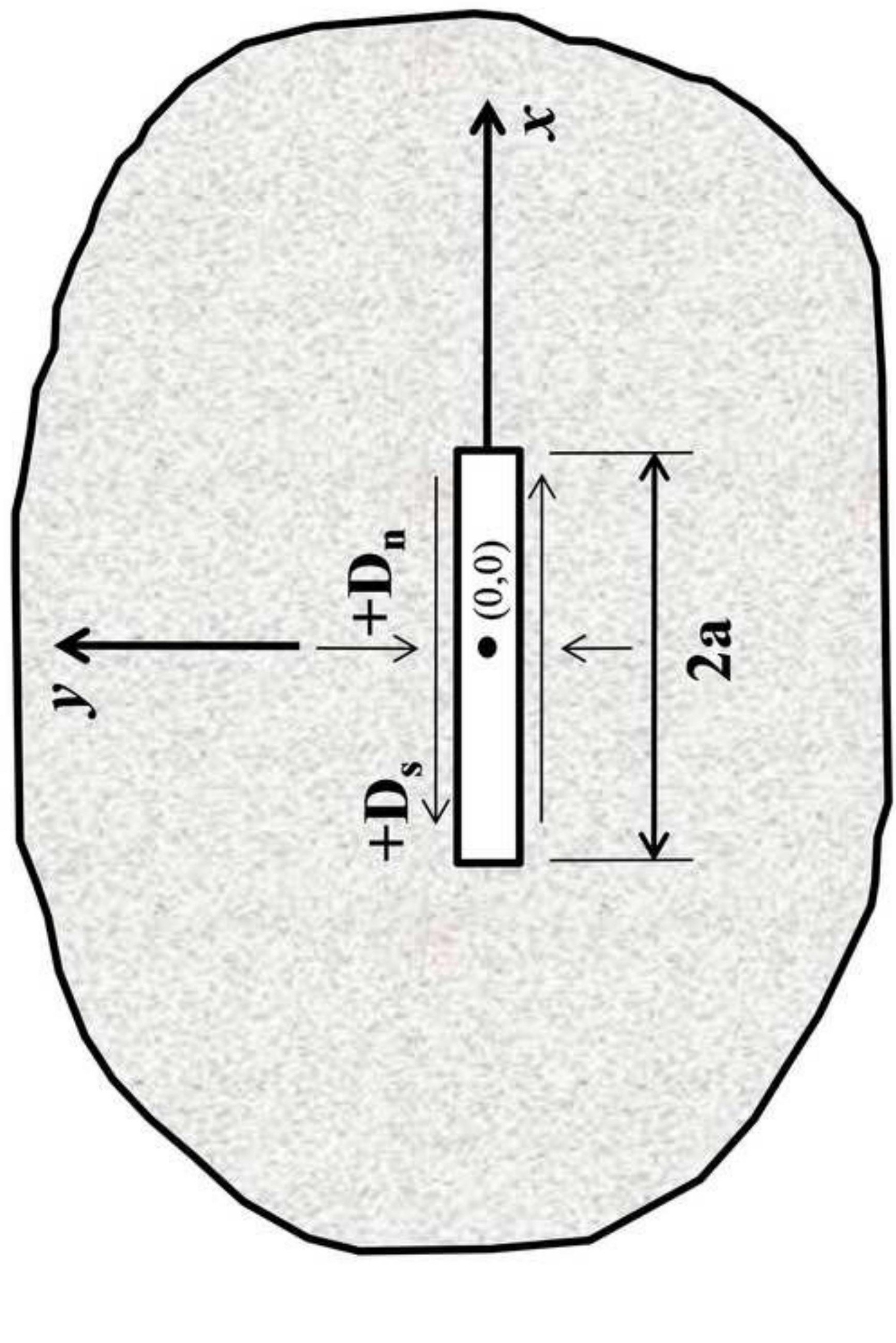



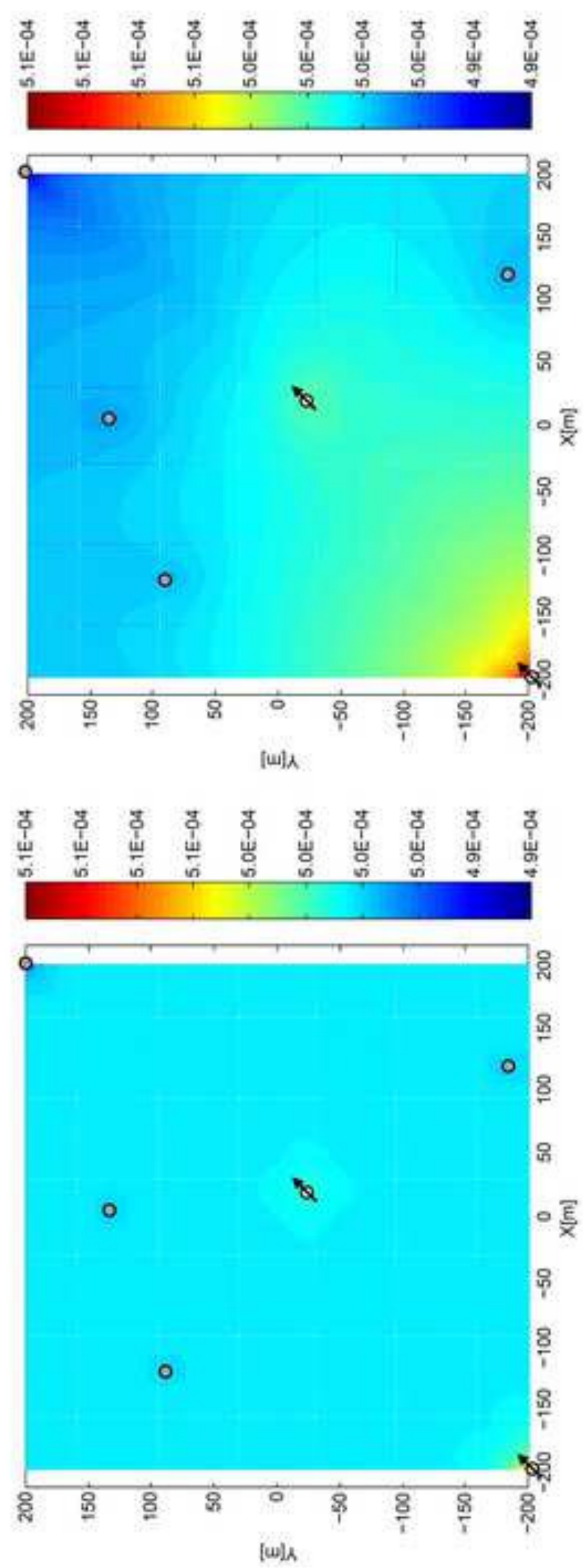

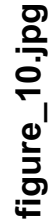



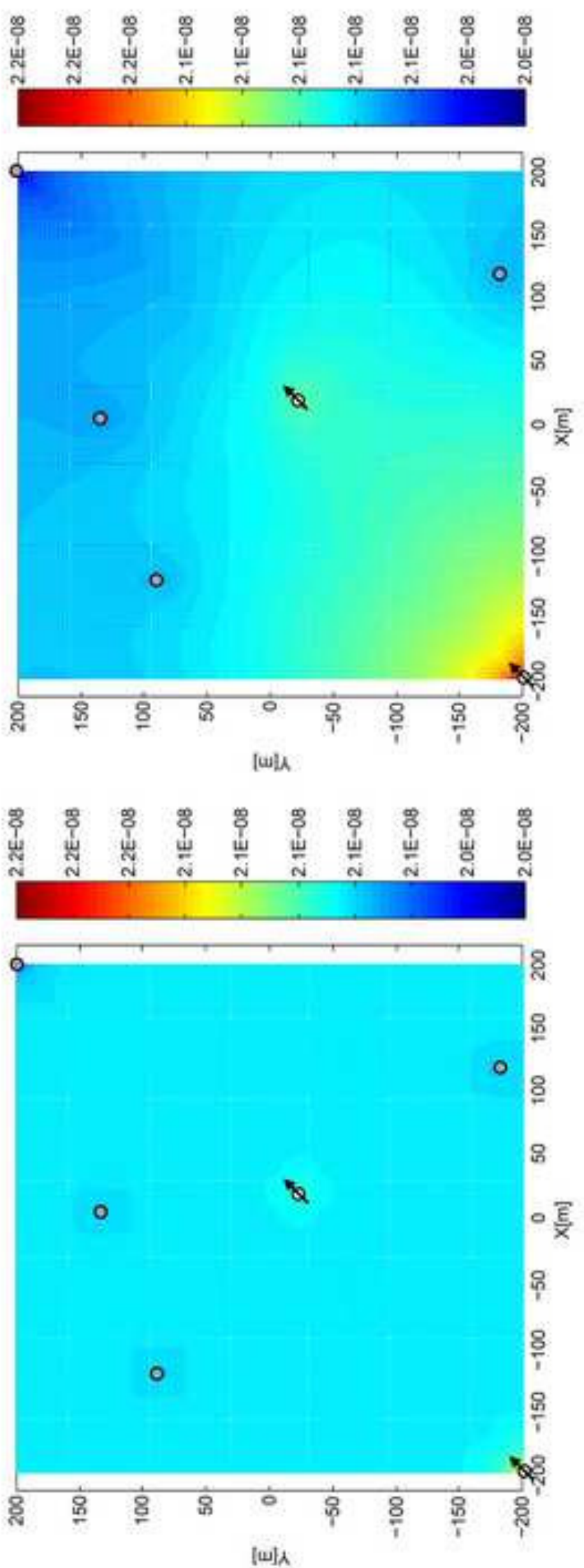

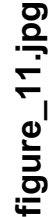



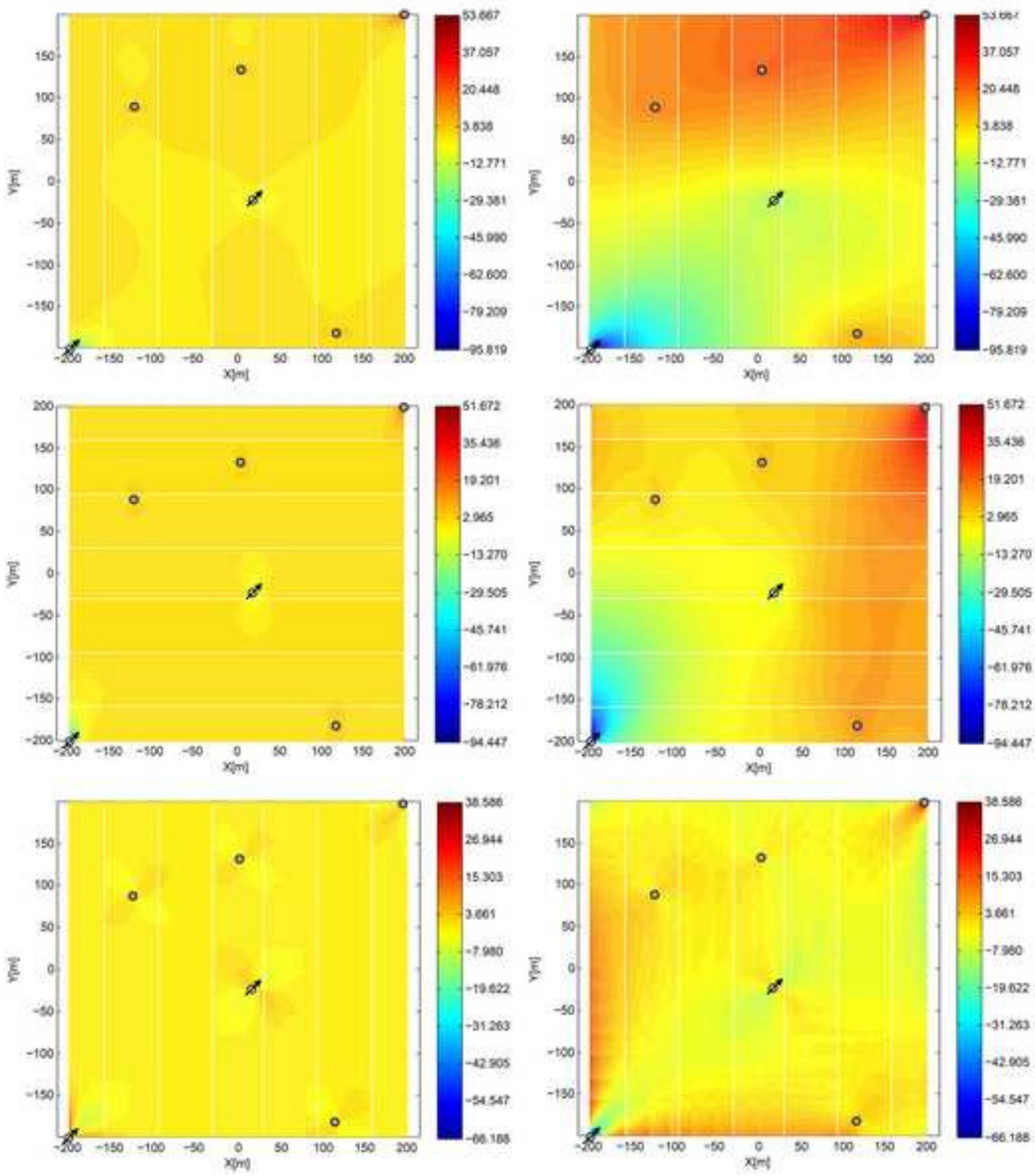